\title{
Partial Regularity of a Generalized Solution to the Navier-Stokes Equations in Exterior Domain
}

\author{
Paolo Maremonti \\ Dipartimento di Matematica ed Applicazioni "R. Caccioppoli," Via Mezzocannone, 8, \\ I-80134 Napoli, Italy
}

\begin{abstract}
In this note, we prove two regularity theorems for solutions to the Navier-Stokes equations of an I.B.V.P. in exterior domains. Namely, we prove that the set $S$ of the singular points of a solution, if not empty, has at most 1Hausdorff measure $H^{1}(S)=0$. Moreover, the set $S$ is enclosed in a sphere of ray $R$ for any $t>0$. These results are obtained as corollaries to the partial regularity results furnished in [2].
\end{abstract}

\section{Introduction}

In this note we study the regularity of suitable solutions to the initial boundary value problem for the nonstationary three-dimensional Navier-Stokes equations in exterior domains. The existence of global-in-time solutions has been proved long ago by Leray and Hopf $[12,14,15]$. They furnish weak solutions of initial boundary value problems. As is well known, two remarkable questions are open about these solutions: the former concerns their uniqueness, the latter their regularity. In this connection, we observe that so far, the regularity of a solution seems to be independent of the regularity of initial data, and recently Scheffer in [27] has proposed a conjecture, namely, that a solution to Navier-Stokes equations exists having an internal singularity, at least in the class of weak solutions verifying only a "generalized energy inequality" as an a priori estimate.

A first result concerning the regularity of weak solutions to the Cauchy problem is due to Leray [15] with his famous "théorème de structure." Later on, the "théorème de structure" was extended to the case of initial boundary value problems $[10,11,19]$. However, we must notice that, so far, in the case of unbounded domains the "théorème de structure" can be obtained for Leray's weak solutions, [8], while for Hopf's weak solutions it is not known, and, on the other hand, we do not know whether Leray's and Hopf's solutions are the same. In [21-26] Scheffer commences and develops an analysis of the set of the possible singular points of a weak solution to the Navier-Stokes equations. Following Caffarelli, Kohn, and Nirenberg [2], a point will be called "singular" for a solution 
$\mathbf{u}$ to the Navier-Stokes equations if and only if solution $\mathbf{u}$ is not essentially bounded on any neighborhood $I(x, t)$ of $(x, t)\left[\right.$ i.e. $\left.\mathbf{u} \notin L^{\infty}(I(x, t))\right]$. In particular, we now quote two papers by Scheffer $[25,26]$. In [25] it is proved, essentially, that the set $S$ of the possible singular points has at most 5/3-Hausdorff measure: the result is proved for a bounded domain $\Omega$ and for the vorticity $\boldsymbol{\omega}=\operatorname{rot} \mathbf{u}$ of solution; however, $\boldsymbol{\omega}$ can be replaced by solution $\mathbf{u}$. In [26], the case of the half-space is considered in $R^{3}$ instead of a bounded domain $\Omega$, and the possibility of singularities on the boundary $x_{3}=0$ is investigated. The set $S$ has in this case at most 1-Hausdorff measure and it is a closed set of the plain $x_{3}=0$. Later on, in [2] the result of [25] for bounded domains was improved and the Cauchy problem was also considered for the Navier-Stokes equations. To this end, in [2], suitable weak solutions are defined, which essentially differ from the usual weak solutions in that they verify a generalized energy inequality [cf. (3.1) below]. However, a higher regularity of the initial data is required in the case of a bounded domain, while for the Cauchy problem it is required only that the initial data is square summable on $R^{3}$. The result about the set $S$ of the possible singular points of solution $\mathbf{u}$ is expressed in terms of a Hausdorff measure constructed on parabolic cylinders, say $P^{k}\left(k \in R^{+}\right)$, and the authors prove that $P^{1}(S)=0$. Further, they consider the question about where the set $S$ of singular points can be possibly localized. This question is tackled for the Cauchy problem. To this aim it is necessary to require an extra condition of summability of initial data. Finally, we must observe that in [2] the above statements are based on local (in space and time) regularity theorems, whose validity is independent of the type of the considered domain and which will be of help also in the note.

In this note we extend to the case of exterior domains some results obtained in [2]. We consider a class of solutions to the Navier-Stokes equations which are more regular than Hopf's solutions and that we call "generalized" for the following reasons. In [8], in a quite different context from the current one, we prove globalin-time existence of solutions to the Navier-Stokes equations corresponding to an initial data $\mathbf{u}^{0} \in J^{9 / 10,5 / 4}(\Omega)$ that, among other things, have generalized derivatives, second-order spatial derivatives and time derivatives, of $5 / 4$ power summability. For the first time and for the Cauchy problem this result was obtained by Ladyzhenskaya [14], and later in [2] for a bounded domain. The properties of the solutions, however, are not sufficient to consider them as regular. Moreover, these solutions do not a priori cover the class of all generalized solutions to the NavierStokes problem, since we have not a uniqueness theorem for them. However, these generalized solutions present the remarkable advantage to inform us about summability properties of the pressure and its gradient, which are necessary to apply our methods to obtain results of partial regularity. The result of this note follows the proof lines of [2]. First, we prove that our generalized solutions verify a generalized energy inequality of the same type as that given in [2]. It allows us to deduce that the set $S$ of singular points, if not empty, at most Hausdorff measure of parabolic type (cf. Definition 1) $P^{1}(S)=0$. Moreover, we prove that, in the further hypothesis of summability of first derivatives in $L^{2}\left(\Omega-S_{R}\right)$, where $\Omega$ is an exterior domain and $S_{R}$ a sphere of ray $R$, the generalized solution is regular at any instant $t>0$, at any point $x \in \Omega$ such that $|x|>R^{\prime}>R$ for a suitable $R^{\prime}$. It is worth noticing that, in order to obtain our results of partial regularity, we do nothing more than 
set ourselves in a position to apply the results of local and partial regularity of [2]. Finally, we observe that by virtue of the higher regularity results due to Serrin [29], we can deduce that the generalized solution belongs to $C^{\infty}\left(\Omega-S_{R}\right)$ and even becomes analytic $[18,32]$.

The plan of the work is as follows. In Sect. 1, after introducing some notation and mathematical preliminaries, we give the statement of our two theorems of partial regularity. In Sect. 2, we recall some results concerning weak and generalized solutions, which in Sect. 3 will be of help for the proof of theorems.

\section{Mathematical Preliminaries and Statement of the Theorems of Partial Regularity}

Let $\Omega$ be a domain of the three-dimensional Euclidean space $R^{3}$, exterior to $v(v \geqq 0)$ compact subregions whose boundaries are supposed $C^{2}$-smooth ${ }^{1}$. We denote by $R_{0}$ the diameter of the complementary $\Omega^{c}$ of $\Omega$ and suppose, without loss of generality, that the origin 0 of coordinates belongs to $\Omega^{c}$. For $\Omega^{\prime} \subseteq \Omega$ and $t>0$ we set $\Omega_{t} \equiv \Omega x(0, t)$. By $L^{p}(\Omega), p \in[1, \infty]$, we denote the Lebesgue space of functions on $\Omega$. Analogous meaning has the symbol $L^{p}\left(\Omega_{t}\right)$. The norm in $L^{p}(\Omega)$ [respectively $L^{p}\left(\Omega_{t}\right)$ ] will be indicated with $|\cdot|_{p}\left(\right.$ respectively $\left.|\cdot|_{p, \Omega_{t}}\right)$. $W^{m, p}(\Omega)$ denotes the usual Sobolev space of order $(m, p)$ of functions on $\Omega$ and $|\cdot|_{m, p}$ is its associated norm. By $W^{r, p}(\Omega)$, with $r \geqq 0$ and $p \geqq 1$, we denote the Sobolev space of functions on $\Omega$ with derivatives of fractional order $(r, p)$ endowed with the intrinsic norm $|\cdot|_{r, p}[1]$. For an open set $A$ in $R^{3}$, we let $C_{0}^{\infty}(A)$ be the set of indefinitely differentiable functions having a compact support in $A$. Moreover, $\mathscr{C}_{0}^{\infty}(\Omega)$ denotes the set of vector valued functions $\Phi$ with components $\Phi_{i} \in C_{0}^{\infty}(\Omega)(i=1,2,3)$ and divergence free. The following completion spaces are considered: $J(\Omega) \equiv$ completion of $\mathscr{C}_{0}^{\infty}(\Omega)$ in $L^{2}(\Omega), J^{1}(\Omega)$ 三completion of $\mathscr{C}_{0}^{\infty}(\Omega)$ in $W^{1,2}(\Omega), \quad J^{2-2 / p}(\Omega) \equiv$ completion of $\mathscr{C}_{0}^{\infty}(\Omega)$ in $W^{2-2 / p, p}(\Omega)$. Finally, by $L^{p}((0, s) ; X)$ we denote the set of functions $u$ from $(0, s)$ into $X$, where $X$ is a Banach space, such that $\int_{0}^{s}|u(\tau)|_{x}^{p} d \tau<+\infty\left(|\cdot|_{x}\right.$ is $X$-norm). For details and properties on the space introduced above, we refer to $[20,30]$.

Definition 1. For any $S \subset R^{3} \times R$ and $k \geqq 0, P^{k}(S)=\sup _{\varepsilon>0} P_{\varepsilon}^{k}(S)$ is said to be a Hausdorff measure of parabolic type, with $P_{\varepsilon}^{k}(S)=\inf \Sigma \delta\left(A_{i}\right)$ where $\left\{A_{i}\right\}_{i \in N}$ is any decomposition of $S$ in a countable number of "parabolic" cylinders with radius $r_{i}$ in space and $r_{i}^{2}$ in time.

If $H^{k}$ is the usual Hausdorff measure, then $H^{k}(S) \leqq C(k) P^{k}(S)$.

The foregoing definition, as well as the Hausdorff measure $H^{k}$, is a particular case of a construction due to Carathéodory; for the basic facts and properties about Hausdorff measure, we erfer the reader to [5]. The measure $P^{k}$ is introduced by Caffarelli et al. in [2], for they found it more suitable to the application of their methods to the Navier-Stokes equations.

We end mathematical preliminaries with some inequalities of Sobolev type.

\footnotetext{
${ }^{1}$ Here the regularity of $\partial \Omega$ is required in order to apply some helpful results, which do not explicitly appear in this note
} 
Lemma 1. Let $f: \Omega \rightarrow R$ with $\nabla f \in L^{p}(\Omega), p<3$. Then there is a constant $f_{0}$ such that $\psi=f-f_{0}$ satisfies the following inequalities:

$$
R^{3-p} \int_{\Gamma}|\psi(R, \gamma)|^{p} d \gamma \leqq C \int_{|x| \geqq R}|\nabla f|^{p} d x, \quad|\psi|_{q} \leqq C|\nabla f|_{p},
$$

where $p \in(1,3), q=3 p /(3-p)$, and $\Gamma \equiv$ angles of spherical polar coordinates in $R^{3}$. Proof. See Lemma 1.3 of [8].

Lemma 2. Let $f \in C_{0}^{\infty}\left(R^{3}\right)$. Then a constant $C$ exists such that

$$
\begin{aligned}
& \left|\left(\varepsilon+|x|^{2}\right)^{\gamma / 2} f(x)\right|_{p} \\
& \quad \leqq C\left|\left(\varepsilon+|x|^{2}\right)^{\alpha / 2} \nabla f(x)\right|_{2}^{a}\left|\left(\varepsilon+|x|^{2}\right)^{\beta / 2} f(x)\right|_{2}^{1-a}, \quad \forall \varepsilon \geqq 0,
\end{aligned}
$$

when

i) $p \geqq 2, \gamma+3 / p>0, \alpha+3 / 2>0, \beta+3 / 2>0$, and $a \in[1 / 2,1]$;

ii) $\gamma+3 / p=a(\alpha+1 / 2)+(1-a)(\beta+3 / 2)$;

iii) $a(\alpha-1)+(1-a) \beta \leqq \gamma \leqq a \alpha+(1-a) \beta$.

Proof. See Lemma 7.1 of [2] or [3].

Finally, the symbol $C$ denotes a generic constant whose numerical value is inessential to our aims, and it may be given several different values in a single computation. For example, we may have in the same line $2 C \leqq C$.

By a generalized solution of the initial boundary value problem of the NavierStokes equations,

$$
\begin{gathered}
\mathbf{u}_{t}+\mathbf{u} \cdot \nabla \mathbf{u}=-\nabla \pi+\Delta \mathbf{u} \quad \text { in } \Omega_{T}, \quad \nabla \cdot \mathbf{u}=0 \text { in } \Omega_{T}, \\
\mathbf{u}(x, 0)=\mathbf{u}_{0} \text { and } \mathbf{u}_{\mid \partial \Omega}=0 \text { with } \mathbf{u}(x, t) \rightarrow 0 \text { for }|x| \rightarrow+\infty,
\end{gathered}
$$

we mean a pair of functions $(\mathbf{u}, \pi)$ defined as follows.

Definition 2. The field $\mathbf{u}: \Omega_{T} \rightarrow R^{3}$ and the scalar function $\pi: \Omega_{T} \rightarrow R(T=+\infty)$ are such that

i) (u, $\pi$ ) satisfies (2.3) almost everywhere in $\Omega_{T}$;

ii) $(\mathbf{u}, \pi)$ satisfies the energy inequality in the following form:

$$
|\mathbf{u}(t)|_{2}^{2}+2 \int_{s}^{t}\left|\nabla^{\mathbf{u}(\tau)}\right|^{2} d \tau \leqq|\mathbf{u}(s)|_{2}^{2}
$$

for $s=0$, for almost all $s>0$ and for all $t \geqq s$;

iii) $\mathbf{u}(x, t)$ can be redefined on a set of zero $t$-measure in such a way that $\lim _{t \rightarrow s^{+}}|\mathbf{u}(t)-\mathbf{u}(s)|_{2}=0$ for all $s \in[0, T)$.

Definition 3. A point $(x, t) \in S \subset \Omega x(0, T)$ is said to be singular for a solution $\mathbf{u}(x, t)$ to Navier-Stokes equations iff $\mathbf{u}(x, t) \notin L^{\infty}(I(x, t))$ for any neighborhood $I(x, t)$ of $(x, t)$; the remaining points, where $\mathbf{u}(x, t) \in L^{\infty}(I(x, t))$ for some neighborhood $I(x, t)$, are called regular.

Remark 1. The set of generalized solutions is certainly non-void. The first result of existence of generalized solutions is due to Ladyzhenskaya for the Cauchy problem [14]. Later, in [2] and [8], the result has been obtained in the case of a 
bounded domain and of an exterior domain, respectively (for exterior domains $\mathrm{cf}$. Lemma 3 below). The generalized solutions have more regularity properties than Hopf's weak solution; in particular, the energy inequality holds in the form ii), which allows to give a "théorème de structure" ${ }^{2}$. Moreover, ii) is interesting in itself from a physical viewpoint and it is necessary for the well-posedness of stability and energy stability study, in the sense of $[13,28]$, in exterior domains: $[6,8,9,11$, 16-17, 19]. However, we prove that any generalized solution (cf. Lemma 3) satisfies a generalized energy inequality (Lemma 6) of the same type of [2], which contains the one referred to in ii) of Definition 2 as a particular case.

Remark 2. From Definition 3 it is clear that a solution $(\mathbf{u}, \pi)$ is regular iff $S=\emptyset$. We observe that by virtue of the higher regularity results due to Serrin [29] we can deduce that $\mathbf{u} \in C^{\infty}(I(x, t))$ and is even analytic $[18,32]$.

Our results concerning the partial regularity of a generalized solution to Navier-Stokes equations in exterior domains are furnished by the following theorems:

Theorem 1. Let $\mathbf{u}_{0} \in J^{9 / 10,5 / 4}(\Omega)$ and $(\mathbf{u}, \pi)$ be a generalized solution obtained in Lemma 3 (below) corresponding to $\mathbf{u}_{0}$. Then the set $S$ of possible singular points has $P^{1}(S)=0$.

Now, we consider the question about where the set $S$ can be possibly localized in $\Omega$. In this connection the following theorem holds:

Theorem 2. Let $(\mathbf{u}, \pi)$ be as in Theorem 1, such that for some $\tilde{R}>0$ $\int_{\Omega-S(0, \bar{R})} \nabla \mathbf{u}_{0}: \nabla \mathbf{u}_{0} d x<\infty$ at initial instant, then there exists a sphere of radius $R$ such that $S \subset S(0, R)$.

Remark 3. In Theorems 1 and 2 we assume $\mathbf{u}(x, 0)=\mathbf{u}_{0} \in J^{9 / 10,5 / 4}(\Omega)$; this hypothesis can be relaxed to $\mathbf{u}_{0} \in Y(\Omega)=\tilde{J}^{2 / 5,5 / 4}(\Omega) \cap \dot{J}(\Omega)$, provided one can prove that $\mathscr{C}_{0}^{\infty}(\Omega)$ is dense in $Y(\Omega)$ endowed with its natural norm ${ }^{3}$.

Theorem 1 is the natural extension of Theorem $\mathrm{A}^{\prime}$ of [2] to the case of exterior domains. However, we actually require $\mathbf{u}_{0} \in J^{9 / 10,5 / 4}(\Omega)$ while in the case of $\Omega$ bounded in [2] it is required only that $\mathbf{u}_{0} \in Y(\Omega)$. Among unbounded domains the Cauchy problem is an exception, where it is sufficient to assume $\mathbf{u}_{0} \in J(\Omega)$.

Theorem 2 is "equivalent" to the corollary (Sect. 8, p. 820) to Theorem D of $[2]$.

\section{Some Preliminary Considerations to the Proof of Theorems}

The following lemma ensures that the class of generalized solutions in exterior domains is non-void

\footnotetext{
${ }^{2}$ For Hopf's solutions we have not a "théorème de structure" in exterior domains, since they a priori do not verify ii). For a more extensive consideration on the question, cf. e.g. [8]

${ }^{3}$ Professor G. Paolo Galdi has recently communicated to the author that such a result of density has been actually obtained by him
} 
Lemma 3. Assume $\mathbf{u}_{0} \in J^{9 / 10,5 / 4}(\Omega)$. Then there exists a generalized solution $(\mathbf{u}, \pi)$ to the system (2.3). In particular, we have:

$$
\begin{gathered}
\int_{0}^{t}\left(|\mathbf{u}(\tau)|_{2,5 / 4}+\left|\mathbf{u}_{\tau}(\tau)\right|_{5 / 4}+|\nabla \pi|_{5 / 4}\right)^{5 / 4} d \tau \leqq C\left\{\left|\mathbf{u}_{0}\right|_{2}^{2}+\left|\mathbf{u}_{0}\right|_{2 / 5,5 / 4}\right\}, \quad \forall t \geqq 0, \\
|\mathbf{u}(t)|_{5 / 4} \leqq\left|\mathbf{u}_{0}\right|_{5 / 4}+C t^{1 / 5}\left|\mathbf{u}_{0}\right|_{2}^{2}, \quad \forall t \geqq 0 .
\end{gathered}
$$

Proof. The existence is proved in [8, Theorem 2.1]; (3.1) is a consequence of (2.6) and (3.2) of Lemma 3.2 of [8].

Lemma 4. Let $(\mathbf{u}, \pi)$ be a generalized solution. Then there exist a set $E \subset(0,+\infty)$ with zero Lebesgue measure and a $\theta \in\left[0, \exp \left(C\left|\mathbf{u}_{0}\right|_{2}^{2}+1\right) C\left|\mathbf{u}_{0}\right|_{2}^{4}\right]$, such that $\forall t_{0} \in(0,+\infty)-E,(\mathbf{u}, \pi)$ is regular in $\left(t_{0}, t_{0}+T\left(t_{0}\right)\right)$, and $(\mathbf{u}, \pi)$ is regular in $(\theta,+\infty)$.

Proof. See Theorem 8 of [11].

Lemma 5. Let $(\mathbf{u}, \pi)$ a suitable weak solution to the system (2.3) in a neighborhood of the point $(x, t)$. If

$$
\limsup _{r \rightarrow 0} r_{t-(7 / 8) r^{2}}^{t+(1 / 8) r^{2}} \int_{|x-y| \leqq r} \nabla \mathbf{u}(y, \tau): \nabla \mathbf{u}(y, \tau) d y d \tau \leqq \varepsilon,
$$

where $\varepsilon$ is a suitable constant, then $(x, t)$ is a regular point of $\mathbf{u}(x, t)$.

Proof. See Proposition 2 of [2].

Remark 4. We stress that $\theta$ in Lemma 4 depends only $\left|\mathbf{u}_{0}\right|_{2}$, but not on the (possibly not unique) particular weak solution considered.

Lemma 5 is stated as in [2]; as a weak solution they mean a weak solution verifying, among other things, a generalized energy inequality of the type (4.1) below.

\section{Proof of the Theorems}

In order to prove Theorem 1, we commence by proving that any generalized solution given by Lemma 3 satisfies a generalized energy inequality, the same proved in [2].

Lemma 6. Let $(\mathbf{u}, \pi)$ be a generalized solution furnished from Lemma 3. Then

$$
\begin{aligned}
& \int_{\Omega}|\mathbf{u}|^{2}(x, t) \Phi(x, t) d x+2 \int_{s}^{t} \int_{\Omega} \nabla \mathbf{u}(x, \tau): \nabla \mathbf{u}(x, \tau) \Phi(x, \tau) d x d \tau \\
& \leqq \int_{\Omega}|\mathbf{u}|^{2}(x, s) \Phi(x, s) d x+\int_{s}^{t} \int_{\Omega}|\mathbf{u}|^{2}(x, \tau)(\Phi,(x, \tau)+\Delta \Phi(x, \tau)) d x d \tau \\
& \quad+\int_{s}^{t} \int_{\Omega}\left(|\mathbf{u}|^{2}(x, \tau)+2 \pi(x, \tau)\right) \mathbf{u}(x, \tau) \cdot \nabla \Phi(x, \tau) d x d \tau,
\end{aligned}
$$

for $s=0$, for almost all $s>0$ and for all $t \geqq s$, for any $\Phi \in C^{\infty}(\Omega x(s, t)) \cap C_{0}(\bar{\Omega})$ such that $\Phi \geqq 0$.

Proof. We give a proof which is quite analogous to that of [2] for the case of $\Omega$ bounded. In [8] the existence of a solution $(\mathbf{u}, \pi)$ is obtained by proving the 
convergence (with respect to suitable norms) of a sequence $\left\{\mathbf{u}_{n}\right\}_{n \in N}$ of solutions to the following system:

$$
\begin{gathered}
\mathbf{u}_{n t}+\mathbf{U}_{n} \cdot \nabla \mathbf{u}_{n}=-\nabla \pi_{n}+\Delta \mathbf{u}_{n} \text { in } \Omega_{T}, \\
\nabla \cdot \mathbf{u}_{n}=0, \quad \mathbf{u}_{n \mid \hat{\alpha} \Omega}=0 \text { and } \mathbf{u}_{n} \rightarrow 0 \text { for }|x| \rightarrow+\infty, \quad \mathbf{u}_{n}(x, 0)=\mathbf{u}_{n}^{0} \in \mathscr{C}_{0}^{\infty}(\Omega),
\end{gathered}
$$

where the functions $\mathbf{u}_{n}$ are defined $\forall t \geqq 0$, these are regular solutions and verify ii) and (3.1)-(3.2); $\mathbf{U}_{n}=\int_{R^{3}} J_{1 / n}(x-y) \mathbf{u}_{n}(y) d y$ and finally $\mathbf{u}_{n}^{0} \rightarrow \mathbf{u}^{0}$ [therefore, in particular, in $J(\Omega)]$. Multiplying both sides in $(*)_{1}$ by $\mathbf{u}_{n} \Phi$ in $L^{2}(\Omega)$ and integrating by parts we have

$$
\begin{gathered}
\int_{\Omega}\left|\mathbf{u}_{n}\right|^{2}(x, t) \Phi(x, t) d x+2 \int_{s}^{t} \int_{\Omega} \nabla \mathbf{u}_{n}(x, \tau): \nabla \mathbf{u}_{n}(x, \tau) \Phi(x, \tau) d x d \tau \\
=\int_{\Omega}\left|\mathbf{u}_{n}\right|^{2}(x, s) \Phi(x, s) d x+\int_{s} \int_{\Omega}\left|\mathbf{u}_{n}\right|^{2}(x, \tau)(\Phi(x, \tau)+\Delta \Phi(x, \tau)) d x d \tau \\
+\int_{s}^{t} \int_{\Omega}\left(\left|\mathbf{u}_{n}\right|^{2}(x, \tau)+2 \pi_{n}(x, \tau)\right) \mathbf{u}_{n}(x, \tau) \cdot \nabla \Phi(x, \tau) d x d \tau, \\
\forall(t, s) \quad \text { such that } t \geqq s \quad \text { and } \quad \Phi \geqq 0 .
\end{gathered}
$$

We observe now that, as $\left\{\mathbf{u}_{n}\right\}$ weakly converges in $L^{2}\left((0, T) ; J^{1}\right)$, by virtue of the Friederichs lemma [4], we have a subsequence $\left\{\mathbf{u}_{n}\right\}$ strongly converging in $L^{2}\left((0, T) ; L^{2}\left(\Omega^{\prime}\right)\right)$ for any compact subdomain $\Omega^{\prime} \subset \Omega$. This last fact implies the existence of another subsequence $\left\{\mathbf{u}_{n}\right\}$ which converges in $L^{2}\left(\Omega^{\prime}\right)$ for almost any $s \in[0, T]$. Moreover, for (2.1) and (3.1), $\pi_{n} \in L^{5 / 4}\left((0, T) ; L^{15 / 7}(\Omega)\right), \forall n \in N$. Here and in the sequel we tacitly suppose that the pressure is $\pi_{n}^{\prime}=\pi_{n}+\pi_{n}^{0}$ : in such a way inequality (2.1) can be applied. However, for the sake of simplicity, the apex' is omitted for $\pi_{n}^{\prime}$. From these considerations and analogous ones for the remaining terms, it is routine to deduce from (3.2) in the limit as $n \rightarrow+\infty,(3.1)$, therefore, the proof is omitted and we refer [14] for details.

From (4.1) and Lemma 5 the proof of Theorem 1 is achieved by a suitable covering of a neighborhood of the possible singular point $(x, t) \in S$ by parabolic cylinders, as is proved in [2] to which we refer for further details of the proof (Sect. 6, p. 806).

To prove Theorem 2 we need the following preliminary lemma.

Lemma 7. In the same hypothesis of Theorem 2, there exists a constant $C_{1}$ for which it is possible to find a sphere centered at 0 and radius $R$, such that for $\left|x_{0}\right| \geqq R$ (with $\left.(R-1)>8 R_{0}\right) L=\int_{\Omega}|\mathbf{u}|^{2}(x, 0)\left|x-x_{0}\right|^{-1} d x<1 / C_{1}=L_{0}$ and for some $\eta>0$,

$$
\int_{\Omega} \frac{|\mathbf{u}(x, t)|^{2} d x}{\left|x-\left(x_{0}+\mathbf{v} t\right)\right|}+\eta \exp \left\{\left(1 / L_{0}\right) \int_{0}^{t} \int_{\Omega} \frac{|\nabla \mathbf{u}(x, \tau)|^{2} d x d \tau}{\left|x-\left(x_{0}+\mathbf{v} \tau\right)\right|}\right\} \leqq L_{0}, \quad \forall t \in\left[0, T_{0}\right]
$$

for any fixed $T_{0}>0,|\mathbf{v}|^{2} t<L_{0}-L$, and $|\mathbf{v}| t \leqq 1$.

Proof. Let $\psi(t)=e^{-k \beta(t)}$ with $\beta(t)=\int_{0}^{t} \int_{\Omega} \frac{\nabla \mathbf{u}(x, \tau): \nabla \mathbf{u}(x, \tau) d x d \tau}{\left[\varepsilon+\left|x-\left(x_{0}+\mathbf{v} \tau\right)\right|^{2}\right]^{1 / 2}}$, where $\varepsilon>0$ and $k>0$ is a constant whose value will be given in the sequel. We consider $g(x, t)$ so defined:

$$
g(x, t)=g_{r}(x) \psi_{h}(t)\left[\varepsilon+\left|x-\left(x_{0}+\mathbf{v} t\right)\right|^{2}\right]^{-1 / 2}=\sigma(\varepsilon, x, t) g_{r}(x) \psi_{h}(t),
$$


where $g_{r}(x) \in C^{\infty}(\Omega)$ is a cut-off function with $g_{r}(x)=1 \forall x$ such that $|x| \leqq r$ and $g_{r}(x)=0$ for $|x| \geqq r+1, \psi_{h}(t)$ is the mollification of $\psi(t)$. By substituting $g$ in (4.1), and taking into account that $\psi$ is absolutely continuous, with $\dot{\psi} \in L^{1}(0, t) \forall t \geqq 0$, in the limit $h \rightarrow 0$ and, subsequently, $r \rightarrow \infty$, it follows

$$
\begin{aligned}
& \psi(t) \alpha(t)+2 \int_{0}^{t} \psi(\tau) \dot{\beta}(\tau) d \tau+\int_{0}^{t} \psi(\tau) \dot{\beta}(\tau) \alpha(\tau) d \tau \\
& \leqq \\
& \\
& \quad+2(0)+\int_{0}^{t} \psi(\tau) \int_{\Omega}^{t}|\mathbf{u}|^{2}(x, \tau)(-\mathbf{v} \cdot \nabla \sigma+\Delta \sigma+\mathbf{u} \cdot \nabla \sigma) d x d \tau \\
& \quad \pi(x, \tau) \mathbf{u} \cdot \nabla \sigma d x d \tau
\end{aligned}
$$

where we have set $\alpha(t)=\int_{\Omega}|\mathbf{u}|^{2}(x, t) \sigma(\varepsilon, x, t) d x$. Now we observe that $\Delta \sigma \leqq 0$ and $|\nabla \sigma| \leqq \sigma^{2} \forall x \in \Omega$. Therefore, it is possible to majorize (4.4) by neglecting the term with $\Delta \sigma$ and by treating the terms in $\nabla \sigma$ with the aid of (2.2) in the following way:

$$
\begin{gathered}
|\mathbf{v}| \int_{\Omega} \sigma^{2}|\mathbf{u}|^{2} d x \leqq C|\mathbf{v}|[\dot{\beta}(t) \alpha(t)]^{1 / 2} \leqq C \dot{\beta}(t) \alpha(t)+|\mathbf{v}|^{2}, \\
\int_{\Omega} \sigma^{2}|\mathbf{u}|^{3} d x \leqq C \dot{\beta}(t) \alpha^{1 / 2}(t) \leqq(1 / 2) \dot{\beta}(t)+C \dot{\beta}(t) \alpha(t),
\end{gathered}
$$

where in the last step we have employed the Cauchy inequality. A different estimate to be found for the pressure. From $(2.3)_{1}$ it follows that $\pi$ obeys the equation

$$
\Delta \pi=-\nabla \mathbf{u}: \nabla \mathbf{u}^{T} \quad \text { almost everywhere in } \Omega \quad\left(\nabla \mathbf{u}^{T} \equiv(\nabla \mathbf{u})^{\text {transpose }}\right) .
$$

Let $\chi(x) \in C^{\infty}(\Omega)$ be a function cut-off such that $\chi(x)=0$ for $|x| \leqq R_{1}$ and $\chi(x)=1$ for $|x| \geqq 2 R$ where $R_{1}=(R-1) / 8>R_{0}$ (for the moment $R>8 R_{0}+1$ is arbitrary). Set $\tau(x)=\pi(x) \cdot \chi(x)$, from (4.7) we have

$$
\Delta \tau(x)=-\nabla \mathbf{u}: \nabla \mathbf{u}^{T} \chi(x)+\pi(x) \Delta \chi(x)+2 \nabla \pi(x) \cdot \nabla \chi(x) \text { in } R^{3} .
$$

Therefore, $\tau(x)=m \Sigma \tau_{i}(x)$ in $R^{3}$ with $m=(\operatorname{meas} S(0,1))^{-1}$ and

$$
\begin{aligned}
& \tau_{1}(x)=\int_{R_{1} \leqq|y| \leqq 2 R_{1}} \frac{\left(u_{i}(y) u_{j}(y) D_{y^{i} y^{j}}^{2} \chi(y)\right) d y}{|x-y|}, \\
& \tau_{2}(x)=2 \int_{R_{1} \leqq|y| \leqq 2 R_{1}} u_{i}(y) u_{j}(y) D_{y^{2}} \chi(y) D_{y^{j}} \frac{1}{|x-y|} d y, \\
& \tau_{3}(x)=\int_{R^{3}} u_{i}(y) u_{j}(y) D_{y^{2} y^{j}}^{2} \frac{1}{|x-y|} \chi(y) d y, \\
& \tau_{4}(x)=\int_{R_{1} \leqq|y| \leqq 2 R_{1}} \frac{(\pi(y) \Delta \chi(y)+2 \nabla \pi(y) \cdot \nabla \chi(y) d y}{|x-y|} .
\end{aligned}
$$

It is easy to verify (cf. for example [7]) that for $|x| \geqq 2 R_{1}, \pi(x) \equiv \tau(x)$. Let us consider the integral with the pressure term, which is majorized by

$$
\begin{aligned}
& \int_{0}^{t} \psi(\tau) \underset{\Omega \cap S\left(0, R_{2}\right)}{\int_{0}^{t}|\pi(x, \tau)||\mathbf{u}(x, \tau)| \sigma^{2}(\varepsilon, x, \tau) d x d \tau} \\
& \quad+\int_{0}^{t} \psi(\tau) \int_{|x| \geqq R_{2}}|\pi(x, \tau)||\mathbf{u}(x, \tau)| \sigma^{2}(\varepsilon, x, \tau) d x d \tau=I_{1}+I_{2},
\end{aligned}
$$


where $R_{2}$ is chosen in such a way that $R_{2} \in\left(2 R_{1},(R-1) / 2\right)$. Since it is

$$
\sigma^{-2}(\varepsilon, x, \tau) \geqq \varepsilon+(1 / 2)\left|x_{0}+\mathbf{v} \tau\right|^{2}-R_{2}^{2} \geqq \varepsilon+(R-1)^{2} / 4 \quad \forall x \in \Omega \cap S\left(0, R_{2}\right),
$$

by employing Hölder's inequality, we have

$$
\begin{aligned}
I_{1} \leqq & C\left[4 \varepsilon+3(R-1)^{2}\right]^{-1} \int_{0}^{t} \psi(\tau)|\pi(\tau)|_{15 / 7}|\mathbf{u}(\tau)|_{15 / 8} d \tau \\
\leqq & C T_{0}^{1 / 5}|\nabla \pi|_{5 / 4, \Omega_{t}}\left(\left|\mathbf{u}_{0}\right|_{5 / 4}+C T_{0}^{1 / 5}\left|\mathbf{u}_{0}\right|_{2}^{2}\right)^{1-\theta}\left|\mathbf{u}_{0}\right|_{2}^{\theta} \\
\leqq & C T_{0}^{1 / 5}\left(\left|\mathbf{u}_{0}\right|_{2}^{2}+\left|\mathbf{u}_{0}\right|_{2 / 5,5 / 4}\right)^{4 / 5} \\
& \times\left(\left|\mathbf{u}_{0}\right|_{5 / 4}+C T_{0}^{1 / 5}\left|\mathbf{u}_{0}\right|_{2}^{2}\right)^{1-\theta}\left|\mathbf{u}_{0}\right|_{2}^{\theta} \quad \forall t \in\left[0, T_{0}\right],
\end{aligned}
$$

where, in the majorization, we have interpolated $L^{15 / 8}(\Omega)$ between $L^{2}(\Omega)$ and $L^{5 / 4}(\Omega), \theta=8 / 9$, and taken into account (2.1) and (3.1)-(3.2). As far as $I_{2}$ concerns, we start with the following position:

$$
I_{2} \leqq C \sum_{h=1}^{4} \int_{0}^{t} \psi(\tau) \int_{|x| \geqq R_{2}}\left|\tau_{h}(x, \tau)\right||\mathbf{u}(x, \tau)| \sigma^{2}(\varepsilon, x, \tau) d x d \tau=\sum_{h=1}^{4} J_{n}
$$

and we estimate the $J_{h}(h=1, \ldots, 4)$ singly. Applying Schwartz' inequality we obtain

$$
\begin{aligned}
J_{1} \leqq & \int_{0}^{t} \psi(\tau)\left(\int_{|x| \geqq R_{2}}\left|\tau_{1}(x, \tau)\right|^{2} \sigma^{2}(\varepsilon, x, \tau) d x d \tau\right)^{1 / 2} \\
& \times\left(\int_{|x| \geqq R_{2}}|\mathbf{u}(x, \tau)|^{2} \sigma^{2}(\varepsilon, x, \tau) d x d \tau\right)^{1 / 2} .
\end{aligned}
$$

By employing Minkowski's generalized inequality, for the first spatial integral, the following estimate holds:

$$
\begin{aligned}
& \int_{|x| \geqq R_{2}}\left|\tau_{1}(x, \tau)\right|^{2} \sigma^{2}(\varepsilon, x, \tau) d x \\
& \quad \leqq\left(\int_{R_{1} \leqq|y| \leqq 2 R_{1}}|\mathbf{u}(y, \tau)|^{2}\left|D^{2} \chi(y)\right|\left(\int_{|x| \geqq R_{2}}|x-y|^{-2} \sigma^{2}(\varepsilon, x, \tau) d x\right)^{1 / 2} d y\right)^{2} \\
& \quad \leqq C\left|\mathbf{u}_{0}\right|_{2}^{4} R_{1}^{-4} \quad \forall \tau \leqq T_{0},
\end{aligned}
$$

where in the last step we have taken into account the property of the cut-off $\chi(y)$ $\left(\left(1 / R_{1}\right)|\nabla \chi(y)|+|\Delta \chi(y)|\right) \leqq C / R_{1}^{2}$ and (2.4). Therefore, employing for the term $\int_{\Omega}|\mathbf{u}(x, \tau)|^{2} \sigma^{2}(\varepsilon, x, \tau) d x,(2.2)$ and subsequently the Cauchy inequality, we have

$$
J_{1} \leqq\left(C T_{0}^{2}\left|\mathbf{u}_{0}\right|_{2}^{8 / 3}\right) / R_{1}^{8 / 3}+\int_{0}^{t} \psi(\tau) \dot{\beta}(\tau) \alpha(\tau) d \tau \quad \forall t \in\left[0, T_{0}\right]
$$

The same arguments can be applied to obtain for $J_{2}$,

$$
J_{2} \leqq\left(C T_{0}^{2}\left|u_{0}\right|_{2}^{8 / 3}\right) / R_{1}^{4 / 3}+\int_{0}^{t} \psi(\tau) \dot{\beta}(\tau) \alpha(\tau) d \tau \quad \forall t \in\left[0, T_{0}\right]
$$

$\tau_{3}$ is a singular integral, then taking into account Calderon-Zigmund's theorem for singular integrals and E. M. Stein's theorem with respect to the measure 
$(\varepsilon+|x|)^{\alpha} d x,[31]$ (Sect. 6.3, p. 49), and employing the Hölder inequality yield

$$
\begin{aligned}
J_{3} & \leqq \int_{0}^{t} \psi(\tau)\left(\int_{\Omega}\left|\tau_{3}(x, \tau)\right|^{3 / 2} \sigma^{2}(\varepsilon, x, \tau) d x\right)^{2 / 3}\left(\int_{\Omega}|\mathbf{u}(x, \tau)|^{3} \sigma^{2}(\varepsilon, x, \tau) d x\right)^{1 / 3} \\
& \leqq C \int_{0}^{t} \psi(\tau) \int_{\Omega}|\mathbf{u}(x, \tau)|^{3} \sigma^{2}(\varepsilon, x, \tau) d x d \tau .
\end{aligned}
$$

Majorizing further on by (2.2) and Cauchy's inequality we arrive at

$$
J_{3} \leqq(1 / 2) \dot{\beta}(t)+C \dot{\beta}(t) \alpha(t) .
$$

Let us consider $J_{4}$. Applying Schwartz's inequality

$$
J_{4} \leqq \int_{0}^{t} \psi(\tau)\left(\int_{|x| \geqq R_{2}}\left|\tau_{4}(x, \tau)\right|^{2} \sigma^{11 / 4}(\varepsilon, x, \tau) d x\right)^{1 / 2}\left(\int_{\Omega}|\mathbf{u}(x, \tau)|^{2} \sigma^{5 / 4}(\varepsilon, x, \tau) d x\right)^{1 / 2} d \tau .
$$

By employing Minkowski's generalized inequality, it follows that

$$
\begin{aligned}
& \int_{|x| \geqq R_{2}}\left|\tau_{4}(x)\right|^{2} \sigma^{11 / 4}(x) d x \\
& \leqq\left(\int_{R_{1} \leqq|y| \leqq 2 R_{1}}\left(|x| \geqq R_{2}|x-y|^{-2} \sigma^{11 / 4}(x) d x\right)^{1 / 2}\left(|\pi(y)|\left|D^{2} \chi(y)\right|+2|\nabla \pi(y)||\nabla \chi(y)| d y\right)\right)^{2} .
\end{aligned}
$$

Since $R_{2}>2 R_{1}$ and the derivatives of $\chi$ have compact support, by taking into account (2.1), we get

$$
J_{4} \leqq C\left(R_{1}\right)^{-2 / 5} \int_{0}^{t} \psi(\tau)|\nabla \pi(\tau)|^{5 / 4}\left(\int_{\Omega}|\mathbf{u}(x, \tau)|^{2} \sigma^{5 / 4}(\varepsilon, x, \tau) d x\right)^{1 / 2} d \tau .
$$

By virtue of Schwartz's inequality applied over and over again, we deduce

$$
\begin{aligned}
\int_{\Omega} \mathbf{u}^{2}(x) \sigma^{5 / 4}(x) d x & \leqq\left(\int_{\Omega} \mathbf{u}^{2}(x) \sigma^{3 / 2}(x) d x\right)^{1 / 2}\left(\int_{\Omega} \mathbf{u}^{2}(x) \sigma(x) d x\right)^{1 / 2} \\
& \leqq\left(\int_{\Omega} \mathbf{u}^{2}(x) \sigma^{2}(x) d x\right)^{1 / 4}\left(\int_{\Omega} \mathbf{u}^{2}(x) \sigma(x) d x\right)^{3 / 4} \\
& \leqq\left(\int_{\Omega} \mathbf{u}^{2}(x) \sigma^{2}(x) d x\right)^{5 / 8}|\mathbf{u}|_{2}^{3 / 4}
\end{aligned}
$$

which for (2.2) and (2.4) it is further on increased by $C\left|\mathbf{u}_{0}\right|_{2}^{3 / 4}(\dot{\beta}(\tau) \alpha(\tau))^{5 / 16}$. Therefore,

$$
\begin{aligned}
J_{4} \leqq & C\left(R_{1}\right)^{-2 / 5}\left|\mathbf{u}_{0}\right|_{2}^{3 / 8} \int_{0}^{t} \psi(\tau)|\nabla \pi(\tau)|_{5 / 4}(\dot{\beta}(\tau) \alpha(\tau))^{5 / 32} d \tau \\
\leqq & C\left(R_{1}\right)^{-64 / 135}\left|\mathbf{u}_{0}\right|_{2}^{4 / 9} T_{0}^{7 / 135}\left(\left|\mathbf{u}_{0}\right|_{2}^{2}+\left|\mathbf{u}_{0}\right|_{2 / 5,5 / 4}\right)^{138 / 135} \\
& +\int_{0}^{t} \psi(\tau) \dot{\beta}(\tau) \alpha(\tau) d \tau \quad \forall t \in\left[0, T_{0}\right],
\end{aligned}
$$

where in the last step we use Cauchy's inequality and take into account (3.1). From (4.4) and (4.5)-(4.14) and taking into account that $8 R_{1}=R-1$, we have

$$
\begin{aligned}
& \psi(t) \alpha(t)+\int_{0}^{t} \psi(\tau) \dot{\beta}(\tau) d \tau+k \int_{0}^{t} \psi(\tau) \dot{\beta}(\tau) \alpha(\tau) d \tau \\
& \leqq \alpha(0)+C_{1} \int_{0}^{t} \psi(\tau) \dot{\beta}(\tau) \alpha(\tau) d \tau+|\mathbf{v}|^{2} t+F\left(T_{0},\left[\mathbf{u}_{0}\right], R, \varepsilon\right) \quad \forall t \in\left[0, T_{0}\right],
\end{aligned}
$$


where the symbol $[\cdot]$ denotes the dependence of $F$ by $\mathbf{u}_{0}$ with respect to suitable norms. Moreover, we recall that $F$ is bounded $\forall \varepsilon>0$ and tends to zero at large $R$. Set $k=C_{1}$ : then (4.15) is equivalent to

$$
\alpha(t)+\exp (k \beta(t))\left(\left(C_{1}^{-1}-\alpha(0)-|\mathbf{v}|^{2} t-F\left(T_{0},\left[\mathbf{u}_{0}\right], R, \varepsilon\right)\right) \leqq C_{1}^{-1} \quad \forall t \in\left[0, T_{0}\right] ;\right.
$$

therefore, if $R$ is chosen in such a way that

$$
\eta=\left(C_{1}^{-1}-\alpha(0)-|\mathbf{v}|^{2} t-F\left(T_{0},\left[u_{0}\right], R, \varepsilon\right)\right)>0,
$$

we have that (4.16) is verified uniformly in $\varepsilon>0$ and $t \leqq T_{0}$. Therefore, we can think of (4.3) as achieved, provided we prove

$$
\mathbf{u}_{0} \in L^{2}(\Omega) \quad \text { and } \int_{\Omega-S(0, \tilde{R})}\left|\nabla \mathbf{u}_{0}(x)\right|^{2} d x<\infty, \text { implies }
$$

$\forall \varepsilon>0, \quad \exists R$ such that $\int_{\Omega} \mathbf{u}_{0}^{2}(x)\left|x-x_{0}\right|^{-1} d x<\varepsilon \quad \forall x_{0}$ such that $\left|x_{0}\right| \geqq R$.

To this aim, set $\tilde{u}=\mathbf{u}_{0} \chi$, where $\chi \in C^{\infty}\left(R^{3}\right)$ is a function such that $\chi(x)=0$ for $|x| \leqq \tilde{R}$ and $\chi(x)=1$ for $|x| \geqq \widetilde{R}+1$. Then $\tilde{u} \in W^{1,2}\left(R^{3}\right)$, and there exists a sequence $\left\{\boldsymbol{\Phi}_{n}\right\}_{n \in N} \leqq C_{0}^{\infty}\left(R^{3}\right)$ such that $\boldsymbol{\Phi}_{n} \rightarrow \tilde{u}$ in $W^{1,2}\left(R^{3}\right)$. Now, we can prove (4.17):

$$
\begin{aligned}
\int_{\Omega} \mathbf{u}_{0}^{2}(x)\left|x-x_{0}\right|^{-1} d x \leqq & \int_{\Omega \cap S(0, \tilde{R}+1)} \mathbf{u}_{0}^{2}(x)\left|x-x_{0}\right|^{-1} d x+\int_{|x| \geqq \tilde{R}} \tilde{u}^{2}(x)\left|x-x_{0}\right|^{-1} d x \\
\leqq & \left(\left|x_{0}\right|-R-1\right)^{-1} \int_{\Omega} \mathbf{u}_{0}^{2}(x) d x+\int_{|x| \geqq \tilde{R}}\left|\Phi_{n}(x)\right|^{2}\left|x-x_{0}\right|^{-1} d x \\
& +\left(\int_{|x| \geqq \tilde{R}}\left|\tilde{u}(x)-\boldsymbol{\Phi}_{n}(x)\right|^{2} d x\right)^{1 / 2} \\
& \times\left(\int_{|x| \geqq \tilde{R}}\left|\nabla\left(\tilde{u}(x)-\boldsymbol{\Phi}_{n}(x)\right)\right|^{2} d x\right)^{1 / 2},
\end{aligned}
$$

and (4.17) follows from this last inequality, since for sufficiently large $n$ and $\left[\left|x_{0}\right|-\operatorname{diam}\left(\operatorname{supp} \Phi_{n}\right)\right]$, the terms on the right side become small. Therefore, the lemma is completely proved.

We are now in a position to prove Theorem 2. The proof follows the lines of the proof given in [2], which we present here for the sake of completeness.

In virtue of Lemma 4, we can restrict ourselves to consider points $(z, t) \in \Omega_{\theta}$. We set $T_{0}=\theta$ in Lemma 7, and let $R$ be the radius of the sphere for which (4.3) holds. Let $\left(z, t^{\prime}\right)$ be a point of $\Omega x\left(0, T_{0}\right)$ such that

$$
\left|z-x_{0}\right|^{2}<\min \left\{t^{\prime}\left(L_{0}-L\right), 1 / 2\right\} \quad \text { for some } x_{0} \text { with }\left|x_{0}\right| \geqq R,
$$

where $L_{0}, L$, and $R$ have the same meaning of the symbols introduced in Lemma 7 . Moreover, we set $\mathbf{v}=\left(z-x_{0}\right) / t^{\prime}$ to obtain

$$
\int_{0}^{t} \int_{\Omega} \frac{|\nabla \mathbf{u}(x, \tau)|^{2}}{\left|x-\left(x_{0}-\mathbf{v} \tau\right)\right|} d x d \tau<+\infty, \quad \text { for } \quad t \in\left(t^{\prime}, \min \left\{t^{\prime 2}\left(L_{0}-L\right) /\left|z-x_{0}\right|^{2}, 2 t^{\prime}\right\} .\right.
$$

For $|z-x| \leqq r$ and $r \in\left(t^{\prime}-(7 / 8) r^{2}, t^{\prime}+(1 / 8) r^{2}\right)$, it follows

$$
\left|x-\left(x_{0}+\mathbf{v} \tau\right)\right| \leqq r+r^{2}|\mathbf{v}|
$$

and we have for $|\mathbf{v}| r \leqq 1$ that

$$
r^{-1} \int_{t^{\prime}-(7 / 8) r^{2}}^{t^{\prime}+(1 / 8) r^{2}} \int_{|z-x| \leqq r}|\nabla \mathbf{u}(x, \tau)|^{2} d x d \tau \leqq 2 \int_{t^{\prime}-(7 / 8) r^{2}}^{t^{\prime}+(1 / 8) r^{2}} \int_{|z-x| \leqq r} \frac{|\nabla \mathbf{u}(x, \tau)|^{2}}{\left|x-\left(x_{0}+\mathbf{v} \tau\right)\right|} d x d \tau .
$$


Since from (4.18) the right-hand is finite $\forall r$, we obtain

$$
\lim _{r \rightarrow 0} r^{-1} \int_{t^{\prime}-(7 / 8) r^{2}}^{t^{\prime}+(1 / 8) r^{2}} \int_{|z-x| \leqq r}|\nabla \mathbf{u}(x, \tau)|^{2} d x d \tau=0,
$$

which, by virtue of Lemma 5 implies the regularity of $\mathbf{u}$ in $\left(z, t^{\prime}\right)$.

Acknowledgements. This work was done under the auspices of GNFM of Italian C.N.R. and on MPI $40 \%$ Contract at the University of Napoli.

\section{References}

1. Adams, R.A.: Sobolev spaces. New York: Academic Press 1975

2. Caffarelli, L., Kohn, R., Nirenberg, L.: Partial regularity of suitable weak solutions of the Navier-Stokes equations. Commun. Pure Appl. Math. 35, 771-831 (1982)

3. Caffarelli, L., Kohn, R., Nirenberg, L.: First-order interpolation inequalities with weights. Compos. Math. 259-275 (1984)

4. Courant, R., Hilbert, D.: Methods of mathematical physics, Vol. 1. New York: Interscience 1953

5. Federer, H.: Geometric measure theory. Berlin, Heidelberg, New York: Springer 1969

6. Galdi, G.P.: Variational methods for stability of fluid motions in unbounded domains. Ric. Mat. 27, 1-8 (1978)

7. Galdi, G.P., Maremonti, P.: A uniqueness theorem for viscous fluid motions in exterior domains. Arch. Ration. Mech. Anal. 91, 375-384 (1986)

8. Galdi, G.P., Maremonti, P.: Monotonic decreasing and asymptotic behavior of the kinetic energy for weak solutions of the Navier-Stokes equations in exterior domains. Arch. Ration. Mech. Anal. (to appear)

9. Galdi, G.P., Rionero, S.: Weighted energy methods in fluid dynamics and elasticity. Lecture Notes in Mathematics, Vol. 1134. Berlin, Heidelberg, New York: Springer 1985

10. Shinbrot, M., Kaniel, S.: The initial value problem for the Navier-Stokes equations. Arch. Ration. Mech. Anal. 21, 270-285 (1966)

11. Heywood, J.G.: The Navier-Stokes equations: on the existence, regularity and decay of solutions. Indiana University Math. J. 29, 639-681 (1980)

12. Hopf, E.: Über die Anfangswertaufgabe für die hydrodynamischen Grundgleichungen. Math. Nachr. 4, 213-231 (1951)

13. Joseph, D.D.: Stability of fluid motion. Berlin, Heidelberg, New York: Springer 1976

14. Ladyzhenskaya, O.A.: The mathematical theory of viscous incompressible flow, 2 nd ed. New York: Gordon and Breach 1969

15. Leray, J.: Sur le mouvement d'un liquide visqueux emplissant l'espace. Acta Math. 63, 193-248 (1934)

16. Maremonti, P.: Asymptotic stability theorems for viscous fluid motions in exterior domains. Rend. Semin. Mat. Univ. Padova 71, 35-72 (1984)

17. Maremonti, P.: Stabilità asintotica in media per moti fluidi viscosi in domini esterni. Ann. Mat. Pura Appl. 142, 57-75 (1985) [brief comm.: On the energy asymptotic decay of viscous incompressible fluid in exterior domains, in proceedings of the workshop on math. aspects of fluid and plasma dynamics, Trieste (1984)]

18. Masuda, K.: On the stability of incompressible viscous fluid motions past objects. J. Math. Soc. Jpn. 27, 294-327 (1975)

19. Masuda, K.: On the analyticity and the unique continuation theorem for solutions of the Navier-Stokes equations. Proc. Jpn. Acad. 43, 827-832 (1967)

20. Miranda, C.: Istituzioni di analisi funzionale lineare, Vols. I-II. Unione Matem. Italiana and C.N.R., Tip Oderisi Ed., Gubbio (1978) 
21. Scheffer, V.: Turbulence and Hausdorff dimension. In: Turbulence and the Navier-Stokes equations. Lectures Notes in Mathematics, Vol. 565. Berlin, Heidelberg, New York: Springer 1976

22. Scheffer, V.: Partial regularity of solutions to the Navier-Stokes equations. Pacific J. Math. 66, 535-552 (1976)

23. Scheffer, V.: Hausdorff measure and the Navier-Stokes equations. Commun. Math. Phys. 55, 97-112 (1977)

24. Scheffer, V.: The Navier-Stokes equations in space dimension four. Commun. Math. Phys. 61, 41-68 (1978)

25. Scheffer, V.: The Navier-Stokes equations on a bounded domain. Commun. Math. Phys. 73, 1-2 (1980)

26. Scheffer, V.: Boundary regularity for the Navier-Stokes equations in half-space. Commun. Math. Phys. 80, 275-299 (1982)

27. Scheffer, V.: A solution to the Navier-Stokes inequality with an internal singularity. Commun. Math. Phys. 101, 47-85 (1985)

28. Serrin, J.: On the stability of viscous fluid motions. Arch. Ration. Mech. Anal. 3, 1-13 (1959)

29. Serrin, J.: The initial value problem for the Navier-Stokes equations. Proc. Symp. Nonlinear Problems. Madison, WI: University of Wisconsin Press 1963, pp. 69-98

30. Solonnikov, V.A.: Estimates for solutions of nonstationary Navier-Stokes equations. J. Sov. Math. 8, 467-528 (1977)

31. Stein, E.: Singular integrals and the differentiability properties of functions. Princeton, NJ: Princeton University Press 1970

32. Kahane, C.: On the spatial analyticity of solutions of the Navier-Stokes equations. Arch. Ration. Mech. Anal. 33, 386-405 (1969)

Communicated by C. H. Taubes

Received July 15, 1986; in revised form October 3, 1986

Note added in proof. Once this work was already accepted for publication, the author was kindly acquainted by Prof. G. P. Galdi with the paper by "H. Sohr and W. von Wahl-A new proof of Leray's structure theorem and smoothness of weak solutions of Navier-Stokes equations for $|x|$ ", where results similar to his are proved. The methods, however, are completely different. 
